\title{
Immunomodulatory Activity of Caesalpinia sappan L. Extracts on Peritoneal Macrophage of Albino Mice.
}

\author{
Sunitha V S $\mathbf{S}^{1}$, Sunil M A ${ }^{2}$, E K Radhakrishnan ${ }^{3}$, Jyothis Mathew ${ }^{4}$ \\ School of Biosciences, Mahatma Gandhi University, Kottayam. Kerala
}

\begin{abstract}
Caesalpinia sappan L. (Caesalpiniaceae) is a medicinal plant which is used as a thirst quencher in southern part of India especially in Kerala. The present study was conducted to scientifically evaluate the effects of extracts of Caesalpinia sappan on phagocytic function of macrophages. In vivo effect of aqueous, ethanol and hexane extract of the plant at two doses (10mg/kg body weight and $25 \mathrm{mg} / \mathrm{kg} \mathrm{b.w.)} \mathrm{were} \mathrm{evaluated} \mathrm{by} \mathrm{oral} \mathrm{administration} \mathrm{of} \mathrm{the} \mathrm{extracts} \mathrm{on} \mathrm{Swiss} \mathrm{albino} \mathrm{male} \mathrm{mice.} \mathrm{In} \mathrm{vitro} \mathrm{immunomodulatory}$ potential of the above extracts at different concentrations $(10 \mu \mathrm{g} / \mathrm{ml}, 25 \mu \mathrm{g} / \mathrm{ml}, 50 \mu \mathrm{g} / \mathrm{ml}$ and $100 \mu \mathrm{g} / \mathrm{ml})$ was studied using peritoneal macrophages from Swiss albino mice. All extracts gave phagocytic modulation in vivo. The ethanol extract of Caesalpinia sappan at a dose of $25 \mathrm{mg} / \mathrm{kg} \mathrm{b.w.} \mathrm{showed} \mathrm{significant}(p<0.05)$ increase in phagocytic activity in comparison with the control. An increased phagocytic response was shown by murine peritoneal macrophages after treatment with the extracts in vitro. A dose dependent response was observed in all cases. The results of the present study indicates the immunomodulatory effect of Caesalpinia sappan extracts on murine peritoneal macrophages, as evidenced by its effect on phagocytosis which is a nonspecific immune mechanism.
\end{abstract}

Keywords: Caesalpinia sappan, phagocytosis, imunomodulation, macrophages, Non specific immune mechanism.

\section{Introduction}

Immunomodulation refers to the suppression or stimulation of immune response. Several plant products and herbal drugs are known to possess immunomodulatory properties. They act by stimulating or suppressing both specific and non specific immunity. Many plants used in traditional medicine have immunomodulating activities. In South India especially in Kerala, water for drinking purpose is boiled along with pieces of roots, leaves or barks of some plants commonly called as thirst quenchers. These plants are reported to have many pharmacological effects. It is a general belief that the water decoctions of thirst quenchers can purify blood and can improve general health. It is also believed that regular consumption of this can improve body's defense mechanism.

Caesalpinia sappan is a species of flowering tree distributed in Asia, including Korea, China, India and Vietnam. It is a folk medicine due to its valuable anti-bacterial, antiinflammatory, emmenagogue and analgesic properties. It is also used for promoting blood circulation and removing stasis. The main component brazilin, is responsible for most of the biological effects of Caesalpinia sappan. Heart wood has been widely used as therapeutics and a colouring agent. The present study was undertaken to assess the immunomodulating activities of extracts from Caesalpenia sappan by analyzing their effects on phagocytosis, using both in vivo and in vitro models.

\section{Materials and Methods}

\subsection{Plant materials}

Heart wood of Caesalpinia sappan was collected in April 2011 from Alappuzha District, Kerala. The specimen was authenticated by Dr. V.T. Antony, Post Graduate \& Research Department of Botany St. Berchmans College,
Changanassery, Kerala. A voucher specimen (No. RHK 6409) has been deposited at Regional Herbarium of Kerala (RHK), St. Berchmans College, Changanassery.

\subsection{Preparation of the Extracts}

Heartwood of the plant was washed, shade dried, powdered and stored in airtight containers. The powder was subjected to soxhlet extraction using solvents of varying polarity, nhexane, ethanol and water. The solvent was removed under reduced pressure and the extract was stored at $4{ }^{\circ} \mathrm{C}$ until use.

\subsection{Animals}

Study was conducted in Swiss albino male mice (20-30g). They were maintained in animal house under standard conditions (temperature $25 \pm 2{ }^{\circ} \mathrm{C}$ ) and fed with standard pellet diet and water ad libitum. This study got clearance from institutional animal ethics committee (Approval number: B21032014-09).

\subsection{Treatment protocol}

The animals were divided into eight groups consisting of six animals each. Group I was of normal control which received phosphate-buffered saline (PBS). Group II received Levamisole at a concentration of $25 \mathrm{mg} / \mathrm{kg}$ body weight (positive control). Animals of group III, IV, V, VI, VII \& VIII received 10 and $25 \mathrm{mg} / \mathrm{kg} \mathrm{b}$. W. of the three extracts of the plant (water, ethanol and hexane extracts dissolved in PBS) orally in volumes of $0.2 \mathrm{ml} /$ day for 30 days.

\subsection{Preparation of peritoneal mouse macrophages}

One milliliter of $3 \%$ Brewer thioglycollate medium (Himedia, India) was injected intraperitoneally into mice as a stimulant to elicit peritoneal macrophages. Four days, later, the peritoneal exudate was collected by peritoneal lavage 


\section{International Journal of Science and Research (IJSR) \\ ISSN (Online): 2319-7064}

Index Copernicus Value (2013): 6.14 | Impact Factor (2014): 5.611

with $10 \mathrm{ml}$ of RPMI-1640 medium (Himedia, India). The exudate was centrifuged at $400 \times \mathrm{g}, 4^{0} \mathrm{c}$ for $10 \mathrm{~min}$. The supernatant was discarded and the cell pellet was resuspended in RPMI 1640 medium. The cell number was determined by counting in a hemocytometer and cell viability was tested by the typan-blue dye exclusion technique (Zhang et al, 2008).

\subsection{In vitro phagocytosis assay}

Phagocytosis assay was performed according to Hay and West Wood (2002) with slight modification. Macrophages $\left(2 \times 10^{6}\right.$ cells $\left./ \mathrm{ml}\right)$ were seeded in $24-w e l l$ plate with a sterile glass cover slip and incubated for $2 \mathrm{~h}$ in $5 \% \mathrm{CO}_{2}$ humidified incubator; then non-adherent cells were removed by washing in RPMI - 1640 medium. The remaining adherent cells were cultured in RPMI - 1640 medium and incubated for $24 \mathrm{~h}$ with different concentration $(10 \mu \mathrm{g} / \mathrm{ml}, 25 \mu \mathrm{g} / \mathrm{ml}$, $50 \mu \mathrm{g} / \mathrm{ml}$ and $100 \mu \mathrm{g} / \mathrm{ml}$ ) of the plant extracts dissolved in $0.1 \%$ DMSO in PBS. Lipopolysaccharide (LPS) at $5 \mu \mathrm{g} / \mathrm{ml}$ was used as mitogen and $0.1 \%$ DMSO in PBS was used as a control. After incubation, the culture medium was removed and the wells were washed with fresh medium. One milliliter RPMI - 1640 medium and $100 \mu \mathrm{g} / \mathrm{ml}$ yeast suspension $\left(10^{8}\right.$ particles $\left./ \mathrm{ml}\right)$ were added to each well and incubated further for $1 \mathrm{hr}$ at $37^{\circ} \mathrm{c}$ in a $5 \% \mathrm{CO}_{2}$ humidified incubator. The wells were washed twice gently with culture medium after incubation. After washing, cover slips were fixed with methanol and then stained with Giemsas dye. The cover slips were removed from the well and inverted on microscope slides and observed under oil immersion (x100) objective. Phagocytic index was calculated by the following equation.

Phagocyte Index $(\mathrm{PI})=$ number of yeast cells phagocytosed by macrophages/no. of macrophages.

\subsection{In vivo phagocytosis assay}

The peritoneal macrophages were collected from treated mice. The remaining procedure was same as that for the in vitro phagocytosis assay.

\subsection{Statistical Analysis}

All the values were expressed as mean \pm SD for six animals. Values for the in vitro assays were expressed as mean $\pm \mathrm{SD}$ for independent experiments. Statistical significance was analysed using analysis of variance (ANOVA) followed by Tukey-Kramer Multiple comparison test. $p$ values less than 0.05 were considered as significant.

\section{Results and Discussion}

Administration of Caesalpinia sappan extracts enhanced the phagocytic activity of murine peritoneal macrophages both in vivo and in vitro. A dose dependent response was observed in vitro and in vivo treatment.

Figure 1: Phagocytosis of yeast cells by murine peritoneal macrophages

Figure 2 shows in vivo effect of Ceasalpinia sappan extract on phagocytic activity of peritoneal macrophages. Ethanol extract treated group exhibited significant phagocytic activity at dose of $25 \mathrm{mg} / \mathrm{kg}$ b.w. when compared with the normal control group. Among others two extracts treated group aqueous extract treated group showed significant $(\mathrm{p}<0.05)$ phagocytic activity over hexane extract treated group.
In in vitro effect of Caesalpinia sappan extracts on phagocytic activity of peritoneal macrophages is showed in Fig 3. All extract showed phagocytic modulation. At $100 \mu \mathrm{g} / \mathrm{ml}$ concentration ethanol extract gave maximum phagocytic index followed by aqueous and hexane extracts. The results of present study shows that Caesalpinia sappan have significant effect on non-specific immune response (phagocytosis). 


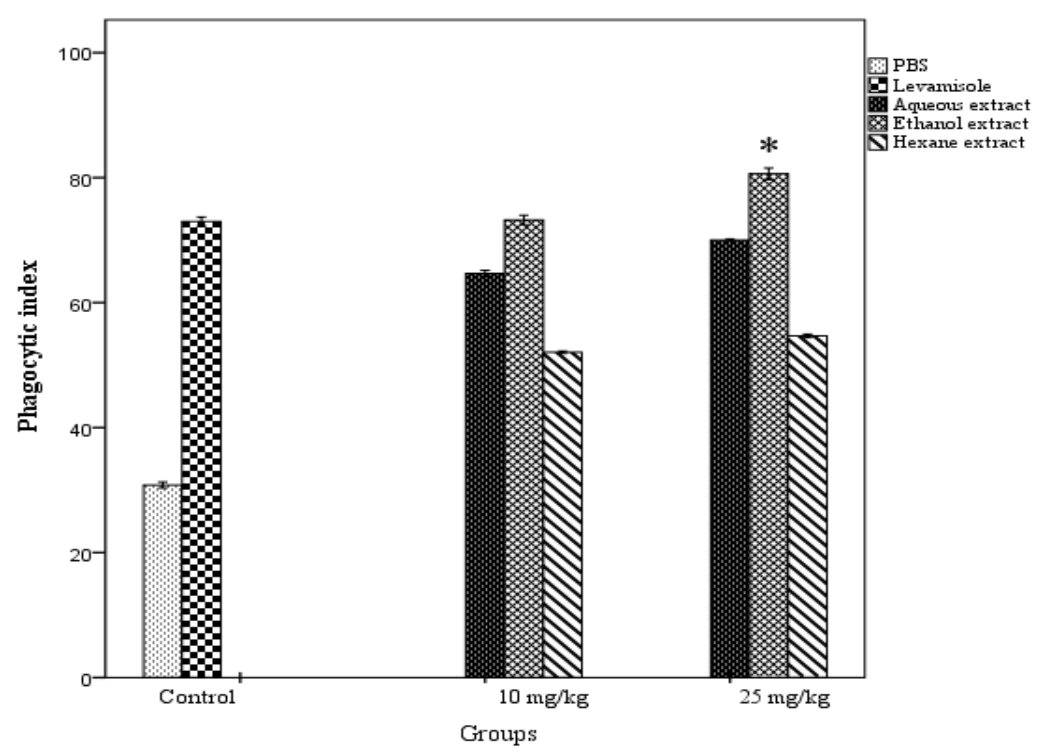

Figure 2: In vivo effect of Caesalpinia sappan extract on phagocytic activity of peritoneal macrophages. ${ }^{*} \mathrm{p}<0.05 v s$.

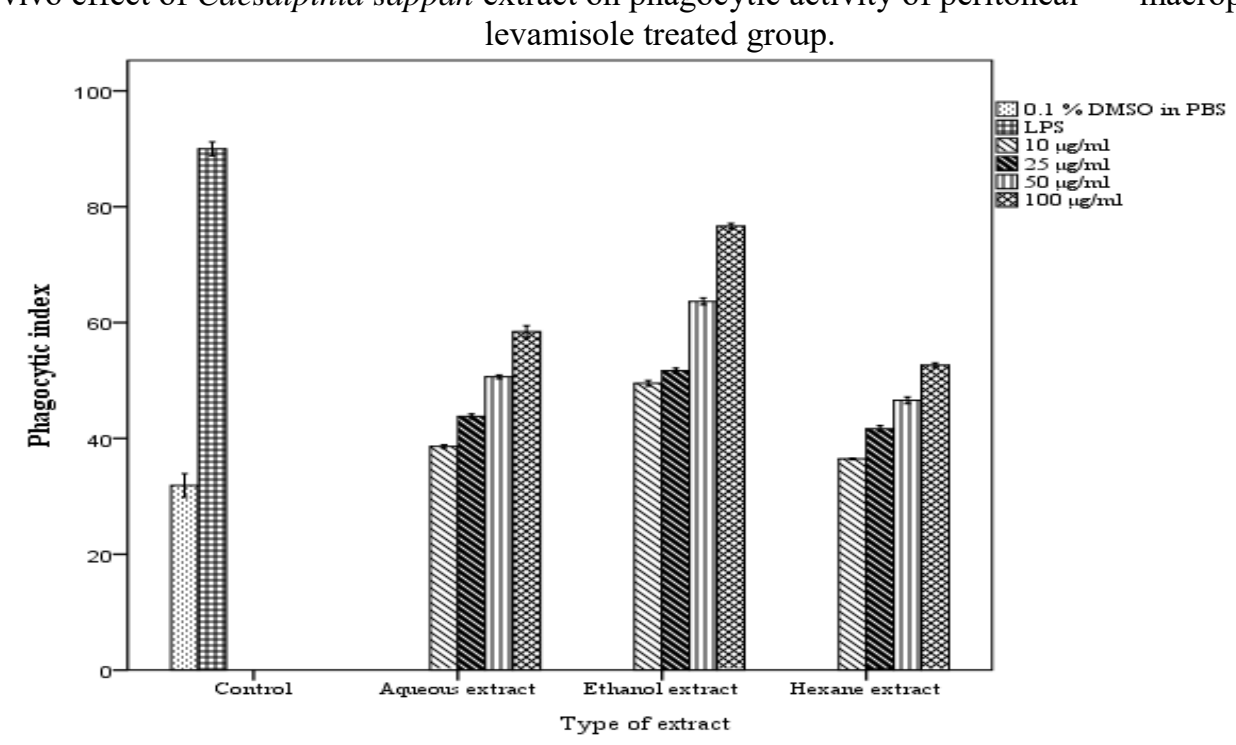

Figure 3: In vitro effect of Caesalpinia sappan extract on phagocytic activity of peritoneal macrophages

Macrophages are mononuclear phagocytes that are widely distributed throughout the body. These cells can contribute to development and homeostasis of innate and adaptive immune response. Macrophages are prodigious secretory cells, and in that role can promote and regulate immune responses and contribute to autoimmune pathologies. Macrophages are highly phagocytic and in this capacity have long been considered to be essential immune effector cells (Zang et al .2008). Macrophages play an important role in inflammation. They have three major functions in inflammation; antigen presentation, phagocytosis and immunomodulation through production of various cytokines and growth factors. Macrophages play a critical role in the initiation, maintenance and resolution of inflammation. Because macrophages produce a wide range of biologically active molecules participated in both beneficial and detrimental outcomes in inflammation, therapeutic intervention targeted macrophages and their products may open new avenues for controlling inflammatory diseases (Fujiwara and Kobayashi, 2005).

\section{Conclusion}

The result of the present study show that Caesalpinia sappan had significant enhancing effect on phagocytic activity of macrophages. Since this effect was observed on oral administration of extract, it can reasonably be assumed that daily consumption of Caesalpinia sappan as thirst quenchers might have some desirable effect on host defense mechanism against infection and inflammatory diseases in which macrophages play important role. Further detailed studies will be helpful in elucidating the mechanism of immune-modulation by this plant. Studies on the effect of extracts of Caesalpinia sappan on the humoral and cellular immune function are in progress in our laboratory.

\section{References}

[1] Anand R. J., Gribar, S. C., Li, J., Kohler J. W., Branca M. F., Dubowski, T., Sodhi, C. P., Hackam, D. J., 2007. Hypoxia causes an increase in phagocytosis by

\section{Volume 4 Issue 12, December 2015}




\section{International Journal of Science and Research (IJSR) \\ ISSN (Online): 2319-7064}

Index Copernicus Value (2013): 6.14 | Impact Factor (2014): 5.611

macrophages in a HIF-1 $\alpha$-dependent manner. Journal of leukocyte biology 82, 1257-1265.

[2] Devis, P.J; Kuttan G.Ethanopharmacology,71,193-200 .doi:so 378-8741(99).2000

[3] G,Mohan et al.,South Asian Journal of Biological Science.1(2)48-57.

[4] Hay, F. C., Westwood O. M. R., 2002. Practical immunology. Blackwell Science Ltd., 203-206.

[5] Ismail, S., Asad, M., 2009. Immunomodulatory activity of Acacia catechu. Indian journal of physiology and pharmacology 53(1), 25-33.

[6] Raynal, a medicinal plant. Journal of ethanopharmacology 140, 239-246.

[7] Saravanan, S., Prakash Babu, N., Pandikumar, P., Raj, M. K., Paulraj, M. G., Ignacimuthu, S., 2012. Immunomodulatory potential of Enicostema axillare (Lam.) A. Williamson E. M., 2002. Major herbs of ayurveda. Churchill livingstone. 13-15.

[8] shrishailappa et.al Biologycal pharma bulletin 26(11)1534-1537.2003

[9] Woosung shin et al.Korean journal of physiology and pharmacology(15)123-128June 2011

[10]Zhang X., Goncalves R., Mosser D. M., 2008. The isolation and characterization of murine macrophages.Current protocols in immunology 83, 14.1.1-14.1.14. 